\title{
Herschel Planetary Nebula Survey (HerPlaNS): Hydrogen Recombination Laser Lines in Mz 3
}

DOI:

10.1093/mnras/sty966

\section{Document Version}

Accepted author manuscript

Link to publication record in Manchester Research Explorer

\section{Citation for published version (APA):}

Aleman, I., Exter, K., Ueta, T., Walton, S., Tielens, A. G. G. M., Zijlstra, A., Montez, R., Abraham, Z., Otsuka, M., Beaklini, P. P. B., van Hoof, P. A. M., Villaver, E., Leal-Ferreira, M. L., Mendoza, E., \& Lepine, J. D. R. (2018). Herschel Planetary Nebula Survey (HerPlaNS): Hydrogen Recombination Laser Lines in Mz 3. Royal Astronomical Society. Monthly Notices, 477(4), 4499-4510. https://doi.org/10.1093/mnras/sty966

\section{Published in:}

Royal Astronomical Society. Monthly Notices

\section{Citing this paper}

Please note that where the full-text provided on Manchester Research Explorer is the Author Accepted Manuscript or Proof version this may differ from the final Published version. If citing, it is advised that you check and use the publisher's definitive version.

\section{General rights}

Copyright and moral rights for the publications made accessible in the Research Explorer are retained by the authors and/or other copyright owners and it is a condition of accessing publications that users recognise and abide by the legal requirements associated with these rights.

\section{Takedown policy}

If you believe that this document breaches copyright please refer to the University of Manchester's Takedown Procedures [http://man.ac.uk/04Y6Bo] or contact uml.scholarlycommunications@manchester.ac.uk providing relevant details, so we can investigate your claim.

\section{OPEN ACCESS}




\title{
Herschel Planetary Nebula Survey (HerPlaNS) ${ }^{\star}$ : Hydrogen Recombination Laser Lines in Mz 3
}

\author{
Isabel Aleman ${ }^{1,2} \uparrow$, Katrina Exter ${ }^{3,4,5}$, Toshiya Ueta ${ }^{6}$, Samuel Walton ${ }^{2,7}$, \\ A. G. G. M. Tielens ${ }^{2}$, Albert Zijlstra ${ }^{8,9}$, Rodolfo Montez Jr. ${ }^{10}$, Zulema Abraham ${ }^{1}$, \\ Masaaki Otsuka ${ }^{11}$, Pedro P. B. Beaklini ${ }^{1}$, Peter A. M. van Hoof ${ }^{12}$, Eva Villaver ${ }^{13}$, \\ Marcelo L. Leal-Ferreira ${ }^{14}$, Edgar Mendoza ${ }^{1}$, Jacques D. R. Lépine ${ }^{1}$ \\ 1 IAG-USP, University of São Paulo, Rua do Matão 1226, Cidade Universitária, 05508-090, São Paulo, SP, Brazil \\ 2 Leiden Observatory, University of Leiden, PO Box 9513, 2300 RA, Leiden, The Netherlands \\ 3 Institute of Astronomy, KU Leuven, Celestijnenlaan 200D, BUS 2401, 3001 Leuven \\ ${ }^{4}$ Herschel Science Centre, European Space Astronomy Centre, ESA, P.O.Box 78, Villanueva de la Cañada, Spain \\ 5 ISDEFE, Beatriz de Bobadilla 3, 28040 Madrid, Spain \\ ${ }^{6}$ Department of Physics and Astronomy, University of Denver, 2112 E. Wesley Ave., Denver, CO 80210, USA \\ 7 Astrophysics Research Institute, Liverpool John Moores University, IC2, Liverpool Science Park, 146 Brownlow Hill, Liverpool L3 5RF, UK \\ 8 Jodrell Bank Centre for Astrophysics, Alan Turing Building, University of Manchester, Manchester, M13 9PL, UK \\ ${ }^{9}$ Department of Physics 83 Laboratory for Space Research, University of Hong Kong, Pok Fu Lam Rd., Hong Kong \\ 10 Smithsonian Astrophysical Observatory, Cambridge, MA 02138, USA \\ 11 Institute of Astronomy and Astrophysics, $11 \mathrm{~F}$ of Astronomy-Mathematics Building, AS/NTU. No.1, Section 4, Roosevelt Rd., \\ Taipei 10617, Taiwan, ROC \\ 12 Royal Observatory of Belgium, Ringlaan 3, B-1180, Brussels, Belgium \\ 13 Departamento de Física Teórica, Universidad Autónoma de Madrid, Cantoblanco, E-28049, Madrid, Spain \\ 14 Argelander-Institut für Astronomie, Universität Bonn, Auf dem Hügel 71, 53121 Bonn, Germany
}

\begin{abstract}
The bipolar nebula Menzel $3(\mathrm{Mz} 3)$ was observed as part of the Herschel Planetary Nebula Survey (HerPlaNS), which used the PACS and SPIRE instruments aboard the Herschel Space Observatory to study a sample of planetary nebulae (PNe). In this paper, one of the series describing $\operatorname{HerPlaNS}$ results, we report the detection of $\mathrm{H}$ I recombination lines (HRLs) in the spectrum of $\mathrm{Mz} 3$. Inspection of the spectrum reveals the presence of $12 \mathrm{HRLs}$ in the 55 to $680 \mu \mathrm{m}$ range covered by the PACS and SPIRE instruments (H11 $\alpha$ to $\mathrm{H} 21 \alpha$ and $\mathrm{H} 14 \beta$ ). The presence of HRLs in this range is unusual for $\mathrm{PNe}$ and has not been reported in $\mathrm{Mz} 3$ before. Our analysis indicates that the HRLs we observed are enhanced by laser effect occurring in the core of $\mathrm{Mz} 3$. Our arguments for this are: (i) the available Mz 3 optical to submillimetre HRL $\alpha$ line intensity ratios are not well reproduced by the spontaneous emission of optically thin ionized gas, as would be typical for nebular gas in PNe; (ii) the compact core of $\mathrm{Mz} 3$ is responsible for a large fraction of the Herschel HRLs emission; (iii) the line intensity ratios for $\mathrm{Mz} 3$ are very similar to those in the core emission of the well known star MWC 349A, where laser effect is responsible for the enhancement of HRLs in the Herschel wavelength range; (iv) the physical characteristics relevant to cause laser effect in the core of MWC 349A are very similar to those in the core of $\mathrm{Mz} 3$.
\end{abstract}

Key words: planetary nebulae: general - planetary nebulae: individual: $\mathrm{Mz} 3$ binaries: symbiotic - stars: emission-line, Be - circumstellar matter - masers

\footnotetext{
* Herschel is an ESA space observatory with science instruments provided by European-led Principal Investigator consortia and with important participation from NASA.

$\dagger$ E-mail: isabel.aleman@usp.br

\section{INTRODUCTION}

Menzel 3 (Mz 3; Ant Nebula; PN 331.7-01.0) is a puzzling object. It has been classified as a planetary nebula (PN), a pre-PN, a symbiotic star, and a symbiotic Mira, but its true 
nature is still not understood (e.g., Cohen et al. 1978; Lopez \& Meaburn 1983; Schmeja \& Kimeswenger 2001; Bains et al. 2004; Cohen et al. 2011). Corradi et al. (2010) placed Mz 3 on the borderline between a symbiotic star and a young PNe, since it has characteristics from both classes of objects.

$\mathrm{Mz} 3$ has a clear bipolar morphology, with a very narrow waist and symmetrically opposed lobes (Cohen et al. 1978). The nebula exhibits multiple outflows and a number of substructures, including a puzzling equatorial ring (Meaburn \& Walsh 1985; Guerrero et al. 2004; Santander-García et al. 2004; Clyne et al. 2015). The outflows along the polar axis can reach hypersonic velocities $\left(\sim 500 \mathrm{~km} \mathrm{~s}^{-1}\right.$; Meaburn \& Walsh 1985; Redman et al. 2000; Santander-García et al. 2004). In the core of the nebula, a disc is obscuring the central source (e.g. Cohen et al. 1978; Kastner et al. 2003; Bains et al. 2004). Chesneau et al. (2007) characterised this disc as rather flat, seen nearly edge-on and rich in amorphous silicates. Spitzer Space Telescope IRAC colours indicate the presence of warm dust at the core of $\mathrm{Mz} 3$ (Cohen et al. 2011). The density in the core exceeds $10^{6} \mathrm{~cm}^{-3}$ (Smith 2003; Zhang \& Liu 2002). Pottasch \& Surendiranath (2005) found that the lobes are composed of ionized gas with $n_{\mathrm{H}} \sim 4 \times$ $10^{3} \mathrm{~cm}^{-3}$, probably ejected in multiple events, and that the ionizing star has $T_{\text {eff }}=39300 \mathrm{~K}$ and $L_{\star}=9100 L_{\odot}$, which are similar to the values obtained by Smith (2003) (30000 K and $10000 \mathrm{~L}_{\odot}$, respectively) using a different procedure. Kastner et al. (2003) detected bright X-ray emission from the core and a possible jet. $\mathrm{Mz} 3$ is also a very bright radio emitter (Bains et al. 2004; Lee et al. 2007). The distance to $\mathrm{Mz} 3$ has been estimated to values between 1.0 and $2.7 \mathrm{kpc}$ (e.g. Cohen et al. 1978; Lopez \& Meaburn 1983; Kingsburgh \& English 1992; Smith 2003; Pottasch \& Surendiranath 2005)

Here, we add a new interesting feature to the list of $\mathrm{Mz} 3$ characteristics by reporting the detection of $\mathrm{HI}$ recombination lines (HRLs) in its Herschel far-infrared (FIR) to submillimetre (submm) spectrum, which we propose is enhanced by laser effect produced in the dense core of this nebula $^{1}$. The study of HRL lasers ${ }^{2}$ is an important tool for inferring physical conditions and kinematics in compact ionized regions, where typical diagnostics, such as forbidden line ratios, are suppressed. As discussed, for example by Hengel \& Kegel (2000) and Strelnitski et al. (1996a), laser effect on HRLs occurs in a somewhat narrow range of physical conditions. Models for the profiles of such lines can provide a detailed view of the physical structure and kinematics, as demonstrated in the studies of Strelnitski et al. (1996b), Martín-Pintado et al. (2011), and Báez-Rubio et al. (2013) of the unresolved core of the B[e] star MWC 349. A detailed discussion of the laser effect on HRL lines and its applications can be found in Strelnitski et al. (1996a).

This paper is organized as follows: Sect. $2 \S 2$ describes the observations and the data reduction method; Sect. 3 presents an overview of the $\mathrm{Mz} 3 \mathrm{FIR} /$ submm spectral fea-

\footnotetext{
${ }^{1}$ It is interesting to note that D. H. Menzel was one of the first scientists to suggest that negative opacities (i.e. light amplification by stimulated emission, later named laser effect) might occur in certain conditions (Menzel 1937) and we now detect laser emission in one of the PNe he discovered (Menzel 1922).

2 For simplification, in the remainder of the text we will use laser to designate the amplification by stimulated emission in all ranges of the electromagnetic spectrum.
}

tures; Sect. 4 presents the detection of FIR/submm HRLs and their characteristics; Sect. 5 discusses the probable laser nature of the hydrogen lines; conclusions are summarized in Sect. 6 .

\section{OBSERVATIONS}

The FIR to submm spectrum of $\mathrm{Mz} 3$ presented in this paper was obtained by the Herschel Planetary Nebulae Survey (HerPlaNS; Ueta et al. 2014). The survey acquired FIR/submm spectra and broadband images of eleven PNe with the PACS (Photodetector Array Camera and Spectrometer; Poglitsch et al. 2010) and SPIRE (Spectral and Photometric Imaging Receiver; Griffin et al. 2010) instruments on board the Herschel Space Observatory (Pilbratt et al. 2010). The resolving power of PACS and SPIRE depend on the wavelength. The resolving power $(\lambda / \Delta \lambda)$ of PACS ranges from 1000 to 5500, while for SPIRE the resolving power ranges from 370 to 1288 . PACS observations were taken for just one pointing toward the centre of the nebulae, while SPIRE observations were made for two pointings, one towards the centre and the other towards the southern lobe of $\mathrm{Mz}$ 3. The PACS spaxels and SPIRE bolometers footprints for each pointing are displayed in Fig. 1.

The data reduction procedure for the HerPlaNS observations is described in Ueta et al. (2014). For PACS data, the reduction was performed with $H_{I P E^{3}}$ (version $11^{4}$, calibration release version 44), using the background normalisation PACS spectroscopy pipeline script and following the procedure described in the PACS Data Reduction Guide: Spectroscopy ${ }^{5}$. For SPIRE, we used HIPE (version 11, calibration tree version 11), following the standard HIPE-SPIRE spectroscopy data reduction pipeline for the single-pointing mode described in the SPIRE Data Reduction Guide ${ }^{6}$.

Line intensities were measured using the code $\mathrm{HERFIT}^{7}$. The code fits the lines with Gaussian profiles. The continuum emission around the line is fitted with a polynomial curve with a user-defined degree. In our case, although a first or second degree polynomial was sufficient for several line fittings, assuming a third-degree polynomial showed the best results for all the lines when compared to SPLAT/STARLINK (Škoda et al. 2014) measurements. HeRFIT is based on the MPFIT algorithm (Markwardt 2009; Moré 1978), which is a widely used algorithm that uses the Levenberg-Marquardt technique to solve the least-squares problem. HERFiT yields

${ }^{3}$ HIPE is a joint development by the Herschel Science Ground Segment Consortium, consisting of ESA, the NASA Herschel Science Center, and the HIFI, PACS and SPIRE consortia (Ott 2010).

${ }_{4}$ Tests we performed showed that the differences in the fluxes found with the more recent version of HIPE (version 15) are within the quoted uncertainties.

5 http://herschel.esac.esa.int/hcss-doc-9.0/load/pacs_ spec/html/pacs_spec.html (Version 1, Aug. 2012)

6 http://herschel.esac.esa.int/hcss-doc-9.0/load/spire_ $\mathrm{drg} / \mathrm{html} / \mathrm{spire} \_\mathrm{drg} . \mathrm{html}$ (Version 2.1, Document Number: SPIRE-RAL-DOC 003248, 06 July 2012)

7 The code, developed by I. Aleman, is available from the author upon request. 

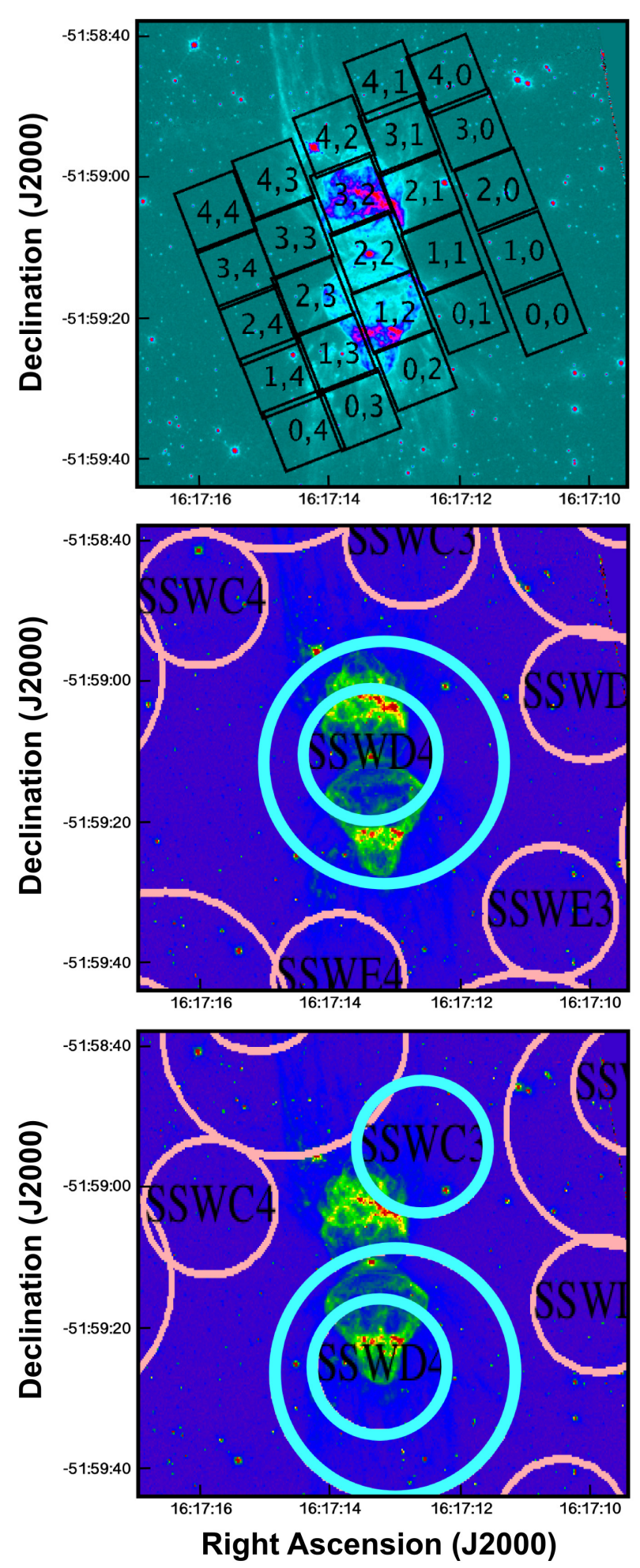

Figure 1. Mz 3 HerPlaNS footprints for PACS (top panel) and SPIRE (centre pointing in the middle and southern lobe pointing in the bottom panel) observations plotted on a WFPC2/HST $\mathrm{Mz} 3$ image using the $\mathrm{H} \alpha-[\mathrm{N}$ II] filter (F658N; GTO program PID 6856, P.I. J. Trauger). The SPIRE bolometers discussed in the text are highlighted in cyan; the others are represented in pink. very similar results to already well established tools such as SPLAT/STARLinK and IRAF/SPLOT ${ }^{8}$.

\section{MZ 3 PACS AND SPIRE SPECTRA}

The full integrated PACS FIR spectrum of Mz 3 is shown in the top panel of Fig. 2. The spectrum was obtained by integrating the flux of spectra extracted from all individual PACS spaxels. By simply summing the spaxel fluxes, we are not considering effects such as the point spread function (PSF) width exceeding the spaxel size. An uncertainty of 30 per cent has been added to the error in the flux measurements to account for this effect.

The bottom panel of Figure 2 shows three SPIRE spectra obtained for $\mathrm{Mz} 3$. From top to bottom, the figure shows the spectrum obtained with the central bolometer for the (i) central and (ii) southern lobe pointings, and (iii) the bolometer SSWC3 for the southern lobe pointing (see Fig.1 for the footprints).

From Fig. 2, it can be seen that the FIR spectrum of $\mathrm{Mz} 3$ is dominated by atomic forbidden lines and a strong continuum due to thermal dust emission. Typical of PNe, the forbidden lines of [N III] $57 \mu \mathrm{m}$, [O I] 63 and $145 \mu \mathrm{m}$, [O III] $88 \mu \mathrm{m},[\mathrm{N}$ II] 122 and $205 \mu \mathrm{m}$, [C II] $157 \mu \mathrm{m}$ are present in the spectra. The [C I] 370 and $609 \mu \mathrm{m}$ lines were not detected.

The surface brightness of individual detected lines are presented in Tables 1 and 2. The first table lists the line surface brightnesses obtained with PACS. Measurements are provided for three distinct regions: (i) all the PACS spaxels, (ii) the combined spaxels $[1,2],[2,2]$ and $[3,2]$, and (iii) the single central spaxel $[2,2]$. The area of each spaxel is $9.4^{\prime \prime} \times 9.4^{\prime \prime}$. The integrated surface brightness measurements from all PACS spaxels are similar to the values obtained by Pottasch \& Surendiranath (2005) and Liu et al. (2001) from the Mz 3 ISO spectrum. The ISO and Herschel/PACS FOV and pointing characteristics are not equal, but both the $I S O$ observations and our PACS spectra cover the lobes and the central region, where most of the forbidden line emission is produced.

The $[\mathrm{N} \mathrm{II}] 205 \mu \mathrm{m}$ line is present in both PACS and SPIRE spectra. The quality of the SPIRE spectrum around $205 \mu \mathrm{m}$ is much better than the corresponding spectral region of the PACS spectrum. The region longwards of 190 microns is not well calibrated in the PACS data produced with HIPE version 11. Therefore we recommend the use of the $[\mathrm{N} \mathrm{II}] 205 \mu \mathrm{m}$ line surface brightness measured from the SPIRE spectrum.

Table 2 presents the measurements from the SPIRE central bolometer for each pointing, i.e. centre and southern lobe. The [N II] $205 \mu \mathrm{m}$ line surface brightnesses measured in each SPIRE pointings are very similar.

For the forbidden lines, between 32 and 49 per cent of the total flux collected by PACS comes from the threespaxel region mentioned above, while between 8 and 23 per cent comes from the central spaxel region. This indicates that the lobes produce a large fraction of the total $\mathrm{Mz} 3$

8 IRAF is distributed by the National Optical Astronomy Observatories, which are operated by the Association of Universities for Research in Astronomy, Inc., under cooperative agreement with the National Science Foundation. 

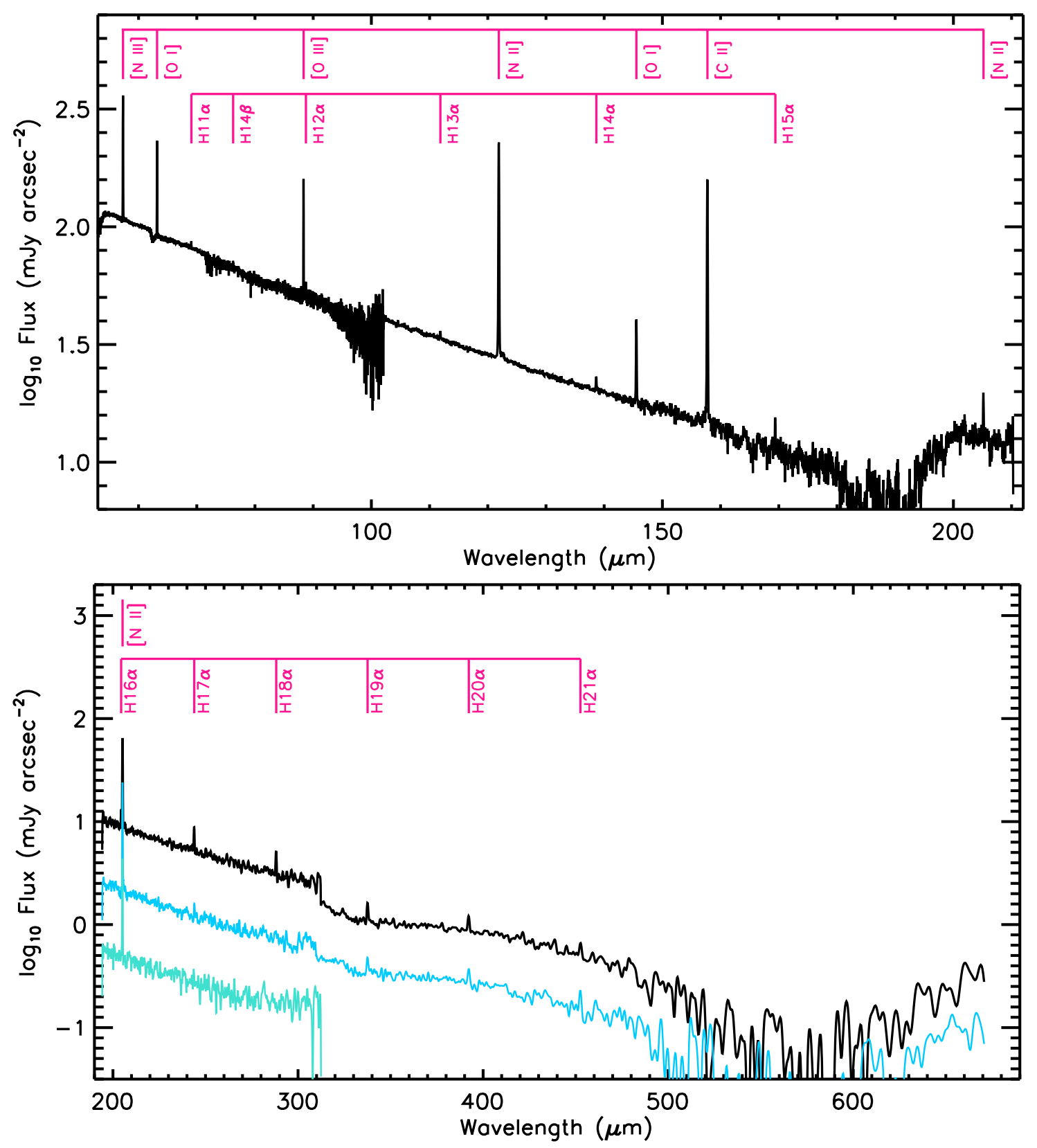

Figure 2. Top panel: Integrated PACS spectrum of Mz 3. The spectrum was obtained by summing the flux of spectra extracted from all individual PACS spaxels. Bottom panel: SPIRE spectra of Mz 3. From the top to bottom, the curves correspond, respectively, to the spectrum from the central bolometers for the central and southern lobe pointings (combined central SSW and SLW bolometers), and the bolometer SSWC3 for the southern lobe pointing. The wavelengths of the atomic forbidden lines, and Hn $\alpha$ and Hn $\beta$ lines detected are indicated.

forbidden line emission. Smith (2003) showed that the emission of some lines, namely [N II] $6583 \AA$, [O II] $3727 \AA$, and [S II] $6717+6731 \AA$, is almost absent in the nucleus. Such emission comes mostly from the lobes, while other lines, such as [O III] $5007 \AA$ and [S III] $9068 \AA$, have important contributions from the two lobes and the core. $\mathrm{H} \alpha$ emission is also found to originate from the core and the two lobes (see Fig. 1 and Smith 2003).

The lines above show that the gas in the lobes is mostly ionized and has an ionization structure that radially extends from the mostly ionized plasma (e.g. [O III]) to a surrounding low-ionization gas (e.g. [O I]). Smith (2003) studied the atomic emission from the lobes and determined their average physical conditions. From the line ratio empirical analysis, he inferred an electron density of $n_{\mathrm{e}} \sim 4500 \mathrm{~cm}^{-3}$ and electronic temperatures in the interval $T_{\mathrm{e}} \sim 7000-15000 \mathrm{~K}$ (depending on the ion used). Similar values were found by Pottasch \& Surendiranath (2005). Using photodissociation region (PDR) line diagnostics diagrams (The PDR Toolbox $^{9}$; Kaufman et al. 2006; Pound \& Wolfire 2008), the forbidden line ratios derived from the measurements in Table 1 (PACS range lines only) provide the following gas densi-

9 http://dustem.astro.umd.edu/pdrt/ 
Table 1. Surface Brightness of the lines detected in the Mz 3 PACS spectrum

\begin{tabular}{|c|c|c|c|c|c|c|c|}
\hline \multicolumn{2}{|c|}{ Line } & \multicolumn{2}{|c|}{ All Spaxels } & \multicolumn{2}{|c|}{ Three Spaxels } & \multicolumn{2}{|c|}{ Central Spaxels } \\
\hline & $\lambda_{0}$ & $\lambda_{\mathrm{obs}}$ & $I$ & $\lambda_{\mathrm{obs}}$ & $I$ & $\lambda_{\mathrm{obs}}$ & $I$ \\
\hline \multicolumn{8}{|c|}{ Forbidden Lines } \\
\hline [N III] & 57.34 & 57.32 & $1543 \pm 482$ & 57.32 & $6205 \pm 1992$ & 57.32 & $4319 \pm 1396$ \\
\hline$[\mathrm{O}$ I] & 63.19 & 63.17 & $651 \pm 205$ & 63.17 & $2654 \pm 835$ & 63.17 & $3745 \pm 1191$ \\
\hline [O III] & 88.36 & 88.34 & $244 \pm 81$ & 88.35 & $860 \pm 282$ & 88.35 & $701 \pm 246$ \\
\hline$[\mathrm{N}$ II $]$ & 121.80 & 121.89 & $591 \pm 186$ & 121.89 & $1706 \pm 550$ & 121.89 & $1495 \pm 483$ \\
\hline$[\mathrm{O}$ I $]$ & 145.54 & 145.52 & $47 \pm 15$ & 145.53 & $166 \pm 54$ & 145.52 & $211 \pm 71$ \\
\hline$[\mathrm{C}$ II $]$ & 157.68 & 157.73 & $257 \pm 85$ & 157.73 & $684 \pm 228$ & 157.73 & $830 \pm 280$ \\
\hline \multicolumn{8}{|c|}{ Hydrogen Recombination Lines } \\
\hline $\mathrm{H} 11 \alpha$ & 69.07 & 69.06 & $14 \pm 6$ & 69.06 & $86 \pm 31$ & 69.06 & $166 \pm 59$ \\
\hline H14 $\beta$ & 76.25 & 76.24 & $20 \pm 10$ & 76.25 & $60 \pm 29$ & 76.24 & {$[42 \pm 26]$} \\
\hline $\mathrm{H} 12 \alpha$ & 88.75 & 88.74 & $7 \pm 4$ & 88.74 & $60 \pm 27$ & 88.74 & $143 \pm 59$ \\
\hline $\mathrm{H} 13 \alpha$ & 111.86 & 111.84 & $8 \pm 3$ & 111.83 & $52 \pm 19$ & 111.83 & $116 \pm 41$ \\
\hline $\mathrm{H} 14 \alpha$ & 138.65 & 138.63 & $8 \pm 3$ & 138.63 & $46 \pm 16$ & 138.63 & $94 \pm 32$ \\
\hline $\mathrm{H} 15 \alpha$ & 169.41 & 169.39 & $5 \pm 3$ & 169.39 & $30 \pm 13$ & 169.39 & $60 \pm 26$ \\
\hline
\end{tabular}

Notes: Rest and observed wavelengths $\left(\lambda_{0}\right.$ and $\left.\lambda_{\mathrm{obs}}\right)$ given in $\mu \mathrm{m}$ and surface brightness (I) in $10^{-17} \mathrm{erg} \mathrm{cm}^{-2} \mathrm{~s}^{-1} \operatorname{arcsec}^{-2}$. Rest wavelengths $\left(\lambda_{0}\right)$ from Kramida et al. (2017).

The H1 $\beta$ central spaxel surface brightness is indicated in brackets to indicate a low signal-to-noise ratio $(\mathrm{S} / \mathrm{N}=2.6)$.

Table 2. Mz 3 lines detected with SPIRE (central bolometer flux only)

\begin{tabular}{|c|c|c|c|c|c|c|}
\hline \multicolumn{2}{|c|}{ Line } & \multicolumn{2}{|c|}{ Centre } & \multicolumn{2}{|c|}{ Southern Lobe } & \multirow{2}{*}{$\begin{array}{l}\text { Beam } \\
\text { FWHM }\end{array}$} \\
\hline Carrier & $\lambda_{0}$ & $\lambda_{\mathrm{obs}}$ & $I$ & $\lambda_{\mathrm{obs}}$ & $I$ & \\
\hline \multicolumn{7}{|c|}{ Forbidden Line } \\
\hline$[\mathrm{N}$ II $]$ & 205.30 & 205.17 & $130 \pm 2$ & 205.16 & $128 \pm 3$ & 17 \\
\hline \multicolumn{7}{|c|}{ Hydrogen Recombination Lines } \\
\hline $\mathrm{H} 16 \alpha$ & 204.41 & 204.38 & $8.9 \pm 0.5$ & 204.43 & $1.2 \pm 0.2$ & 17 \\
\hline $\mathrm{H} 17 \alpha$ & 243.93 & 243.86 & $8.3 \pm 0.4$ & 244.02 & $3.1 \pm 0.3$ & 17 \\
\hline $\mathrm{H} 18 \alpha$ & 288.23 & 288.14 & $4.6 \pm 0.2$ & 288.03 & $2.7 \pm 0.3$ & 19 \\
\hline $\mathrm{H} 19 \alpha$ & 337.59 & 337.57 & $1.16 \pm 0.06$ & 337.56 & $0.99 \pm 0.05$ & 36 \\
\hline $\mathrm{H} 20 \alpha$ & 392.28 & 392.16 & $0.73 \pm 0.02$ & 392.25 & $0.72 \pm 0.05$ & 34 \\
\hline $\mathrm{H} 21 \alpha$ & 452.58 & 452.65 & $0.33 \pm 0.02$ & 452.47 & $0.44 \pm 0.06$ & 30 \\
\hline
\end{tabular}

Notes: Rest and observed wavelengths $\left(\lambda_{0}\right.$ and $\left.\lambda_{\text {obs }}\right)$ given in $\mu \mathrm{m}$, surface brightness in $10^{-17} \mathrm{erg} \mathrm{cm}^{-2} \mathrm{~s}^{-1} \operatorname{arcsec}^{-2}$, and beam full width at half maximum (FWHM) in arcsec (Swinyard et al. 2010). Rest wavelengths from Kramida et al. (2017).

ties $\left(n_{\mathrm{H}}\right)$ and incident fluxes $\left(G_{0}\right.$, the incident flux in Habing units, i.e., relative to the average interstellar medium flux of $1.6 \times 10^{-3} \mathrm{ergs} \mathrm{cm}^{-2} \mathrm{~s}^{-1}$; Pound \& Wolfire 2008): $n_{\mathrm{H}}=1800$, $5600,5600 \mathrm{~cm}^{-3}$ and $G_{0}=3200,1000,1800$ for, respectively, all spaxels, the three central spaxels, and the central spaxel measurements. A detailed pan-chromatic photoionization model (e.g. Otsuka et al. 2017) of Mz 3 will be presented in a upcoming HerPlaNS paper.

No molecular line emission was detected in our data set. The absence of $\mathrm{CO}$ emission in our spectra, even from the core spaxel, where a dense disc/torus is present, is noteworthy. Weak emission of CO $J=2-1$ was detected by Bujarrabal \& Bachiller (1991), indicating a total molecular mass of $1.7 \times 10^{-3} M_{\odot}$, which corresponds to a fraction of $1.3 \times 10^{-2}$ of the ionized mass, according to Huggins et al. (1996). Although the detection of $\mathrm{CO}$ has been reported, no $\mathrm{H}_{2}$ rovibrational lines are present in the near-IR spectrum obtained by Smith (2003) and no rotational $\mathrm{H}_{2}$ lines are present in the ISO spectra published by Pottasch \& Surendiranath (2005).

The most striking feature in the $\mathrm{Mz} 3$ Herschel spectrum is, however, the presence of HRLs, which we discuss in the next section.

\section{H I RECOMBINATION LINES IN MZ 3}

We detected hydrogen recombination $\alpha$ lines from $\mathrm{H} 11 \alpha$ to $\mathrm{H} 21 \alpha$ in the Herschel PACS and SPIRE spectra of Mz 3 (Fig. 2). Only one $\beta$ HRL was detected in the range: H14 $\beta$.

To the best of our knowledge, no detection of HRLs in radio frequencies has been reported for $\mathrm{Mz} 3$ thus far. In previous studies of this nebula with the ISO mid-infrared (MIR) spectrum, the $\mathrm{H} 4 \alpha(n=5 \rightarrow 44.05 \mu \mathrm{m})$ and $\mathrm{H} 5 \alpha(n=6 \rightarrow 5$ $7.46 \mu \mathrm{m}$ ) lines were detected, but no lines from higher $n$ levels were seen (Sloan et al. 2003; Pottasch \& Surendiranath 
2005). Many lines from the hydrogen series have been detected in the optical range (Zhang \& Liu 2002; Smith 2003).

Surface brightnesses for the HRLs observed with Herschel are listed in Tables 1 and 2. As described in the previous section, the first table shows the line surface brightnesses obtained with PACS, while the second presents the measurements with the SPIRE central bolometer for the two available pointings (centre and southern lobe). H16 $\alpha$ at 204 $\mu \mathrm{m}$ was only detected with SPIRE.

Figures 3 to 5 show the PACS spectra for each spaxel zoomed in around the lines detected in the nebula. The $\mathrm{H} n \alpha$ recombination lines detected with Herschel are mostly produced in the central region of $\mathrm{Mz} 3$, while the $\mathrm{C}, \mathrm{N}$, and $\mathrm{O}$ forbidden lines, a significant fraction is produced in the lobes. The central spaxel, with some contribution from the neighbour spaxels $[1,2]$ and $[3,2]$ (each covering part of one of the $\mathrm{Mz} 3$ lobes; see Fig. 1), dominates the emission of the HRLs. Intense emission of forbidden lines present typically in the ionized region (e.g. [O III] $88 \mu \mathrm{m}$ and [N II] $122 \mu \mathrm{m}$ lines) is much more extended than the emission of the FIR $\mathrm{H} n \alpha$ lines in Mz 3. From Table 1, we see that the HRLs surface brightnesses of the central spaxel tend to be higher than those of the spectra integrated over the central three spaxels and over all the spaxels, indicating that the emission is highest close to the centre. For the forbidden lines, the central spaxel surface brightness is comparable (within uncertainties) to the value obtained from the integrated central three spaxels, but much smaller than when all the spaxels are integrated.

The H14 $\beta$ line at $76 \mu \mathrm{m}$ is very faint and could not be detected above the three-sigma limit in individual spaxels. The line is, however, detected in the integrated spectra (all and three central spaxels).

It is interesting to compare the relative intensity of the of the PACS FIR HRLs at different positions in the nebula with those of the optical HRLs. Zhang \& Liu (2002) measured the $\mathrm{H} \beta$ emission in a slit positioned along the polar symmetry axis of the nebula, showing that the profile has three peaks, one in the core and one in each lobe. The core emission corresponds to $\sim 45$ per cent of the total $\mathrm{H} \beta$ emission. In our measurements of the H15 $\alpha$ line at $139 \mu \mathrm{m}$, for example, the central spaxel emission corresponds to 47 per cent of the total PACS emission for this line. For other FIR $\mathrm{H}$ I lines, the central spaxel emission can account for 45 to 84 per cent of the total PACS emission. If we consider not only the central spaxel, but also spaxels $[1,2]$ and $[3,2]$, the emission corresponds to 70 per cent of the total PACS emission of $\mathrm{H} 15 \alpha$ and 68 to 100 per cent for the other $\mathrm{H} n \alpha$ lines. As a comparison, the fraction of the forbidden line emission from the central spaxel mentioned in the previous section ranges from 10 to 23 per cent and for the three central spaxels ranges from 32 to 49 per cent of the total PACS flux. For H14 $\alpha$ line the fractions are 8 and 36 per cent for the central and three central spaxels, respectively.

From all the SPIRE bolometers, for both pointings, only the central bolometer (see footprints in Fig 1) shows HRL emission. For the centre pointing, the central bolometer covers the core and and part of the bipolar lobes depending on the wavelength, since the SPIRE bolometer beam size depends on the wavelength. In Fig 1, the size of the two circles representing the central bolometers indicates approximately the size of the beam for the smallest and largest possible SPIRE beams.

For the lobe pointing, the central bolometer observation covers part of the southern lobe and may include part of the central core depending on the wavelength observed. Figure 6 shows the beam sizes for each Hn $\alpha$ line observed in our spectra (Makiwa et al. 2013). For shorter wavelengths, the central bolometer spectra should be dominated by the southern lobe emission.

In Fig. 2, we also show the SSWC3 bolometer spectrum for the lobe pointing, which only covers short wavelengths. The bolometer covers the northern part of the northern lobe and does not show any evidence of HRL emission.

\section{EVIDENCE OF LASER EFFECT}

The detection of FIR/submm HRLs is unusual. The detection of HRLs in the FIR/submm spectrum of $\mathrm{Mz} 3$ was not initially expected as in the range of physical conditions typical for PNe such lines should be much fainter than the atomic forbidden lines. Hydrogen recombination lines were not detected in any of the other 10 PNe observed in HerPlaNS (NGC 40, NGC 2392, NGC 3242, NGC 6445, NGC 6543, NGC 6720, NGC 6781, NGC 6826, NGC 7009, and NGC 7026). A search in the literature revealed that FIR/submm HRLs have been observed in only a few PNe. Such lines have been reported in the young PN NGC 7027 by Liu et al. (1996) and Wesson et al. (2010) and in the prePNe AFGL 2688 and AFGL 618 by Wesson et al. (2010). The identification of $\mathrm{H} 12 \alpha$ and $\mathrm{H} 13 \alpha$ made by Liu et al. (1996) in the ISO FIR spectrum of the young PN NGC 7027 is questionable according to the authors themselves. However, Wesson et al. (2010) reported the detection of a few HRLs in the SPIRE spectra of NGC 7027.

The spontaneous emission of an optically thin gas does not fit the Mz $3 \mathrm{HRL}$ ratios. The plots in Fig. 7 show the Herschel PACS and SPIRE HRL fluxes relative to $\mathrm{H} 11 \alpha$ plotted as a function of $n$ for $\mathrm{Mz} 3$ (dots). The top panel in Figure 7 uses the SPIRE centre pointing, while the bottom plot uses the southern lobe pointing.

As mentioned in the previous section, the SPIRE beam depends on the wavelength. Moreover the SPIRE beam is different from the PACS field of view (FOV). Also in the previous section, we note that most of the emission comes from the region covered by the three central spaxels $[1,2]$, $[2,2]$, and [3,2]. Zhang \& Liu (2002) and Redman et al. (2000) showed, respectively, that most of the $\mathrm{H} \beta$ emission (detected within a slit across the nebular major axis) and most of the $\mathrm{H} \alpha^{10}$ emission are produced in a region within $15^{\prime \prime}$ of the central source. Therefore it is natural to assume that the PACS three central spaxels region mentioned above and the SPIRE central bolometer for central pointing in any wavelength cover most of the HRL emission. Thus no beam corrections need to be applied for the comparison in Fig. 7 top. The case of SPIRE lobe pointing will be discussed later in this section.

10 Note that here we are using the regular way of representing the $\alpha$ and $\beta$ lines for the Balmer series, i.e. omitting the number 2 for the lower $n$ level of the series 

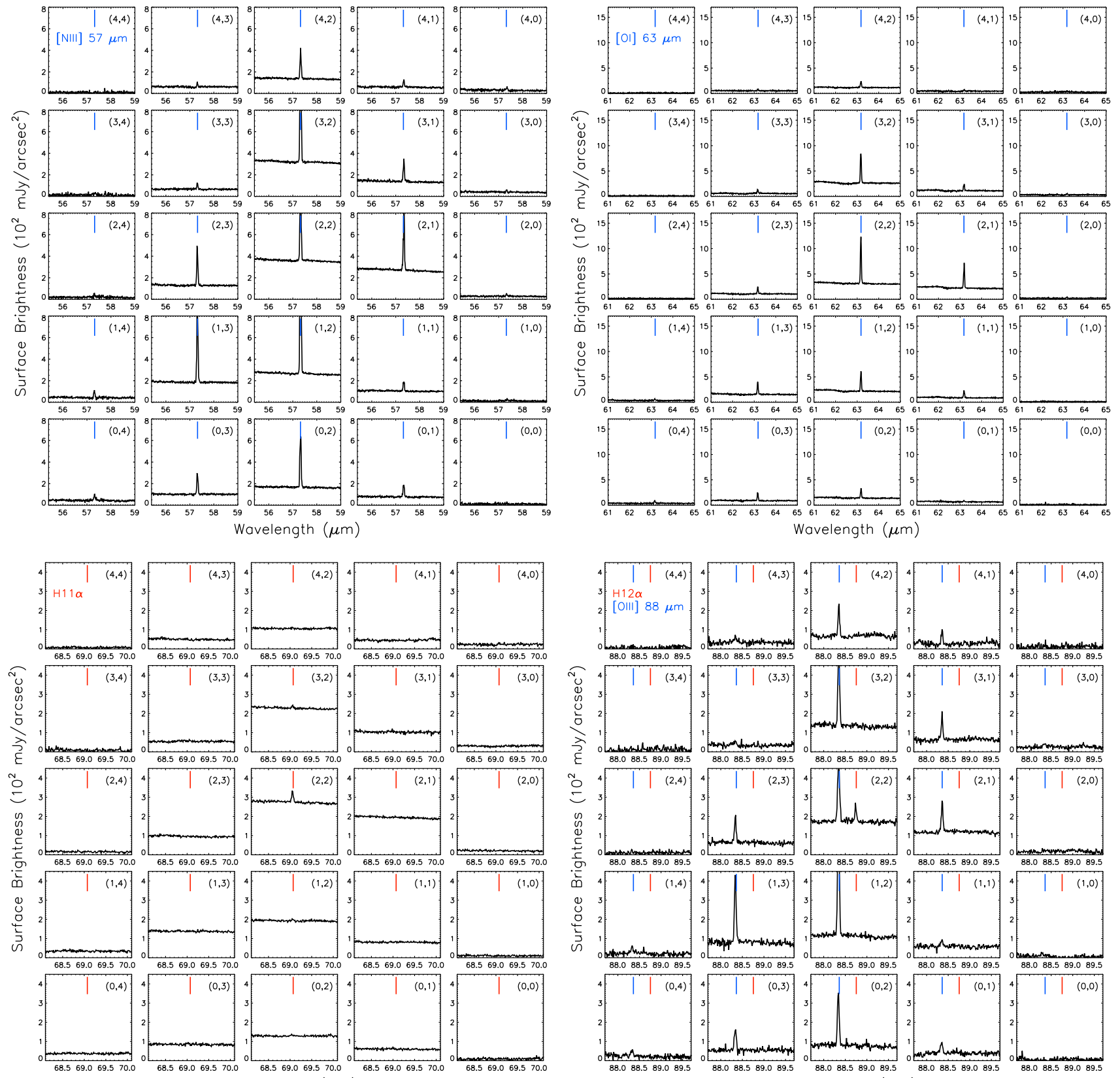
Wavelength $(\mu \mathrm{m})$ Wavelength $(\mu \mathrm{m})$

Figure 3. Spatial distribution of lines detected in the Herschel/PACS FIR spectrum of Mz 3. The coordinates of the corresponding spaxel are indicated in the top right corner of each panel. The coordinates correspond to those indicated in the PACS footprint displayed in Fig. 1.

In Fig. 7, we also included ISO $\mathrm{MIR}^{11}$ and optical data. The ISO $\alpha$ lines included are $\mathrm{H} 4 \alpha$ (Bracket series) and $\mathrm{H} 5 \alpha$ (Pfund) and the optical is $\mathrm{H} \alpha$ (Balmer). Since the ISO FOV of $14^{\prime \prime} \times 20^{\prime \prime}$ (which is centred on the central source with the large dimension along the main nebular axis) should also include most of the HRL emission, no correction of the fluxes to account for different beams/FOV is required. $\mathrm{H} \alpha$ to $\mathrm{H} \beta$ ratios have been re-

11 Our measurements were taken from the spectra obtained from the ISO SWS Atlas constructed by Sloan et al. (2003). For these lines, we used SPLAT/STARLink to make the measurements. ported by Zhang \& Liu (2002) $(\mathrm{H} \alpha / \mathrm{H} \beta=3.26)$ and Smith (2003) $(\mathrm{H} \alpha / \mathrm{H} \beta=5.10)$. The difference between the ratios is due to different de-reddening methodologies. The uncorrected ratios obtained by each group are very similar despite using different instrumentation and FOV. To calculate the $\mathrm{H} \alpha$ absolute flux, we assume the $\mathrm{H} \beta$ flux estimated from radio emission at $6 \mathrm{~cm}$ by Pottasch \& Surendiranath (2005), $F_{\mathrm{H} \beta}=2.86 \times 10^{-10} \mathrm{erg} \mathrm{cm}^{-2} \mathrm{~s}^{-1}$. The gas at such frequency seems to be optically thin, while there is evidence that the direct measure of $\mathrm{H} \beta$ is probing only the outer gas layers Pottasch \& Surendiranath (2005). This is important to consider as the FIR lines probe regions deep into the dense core, 

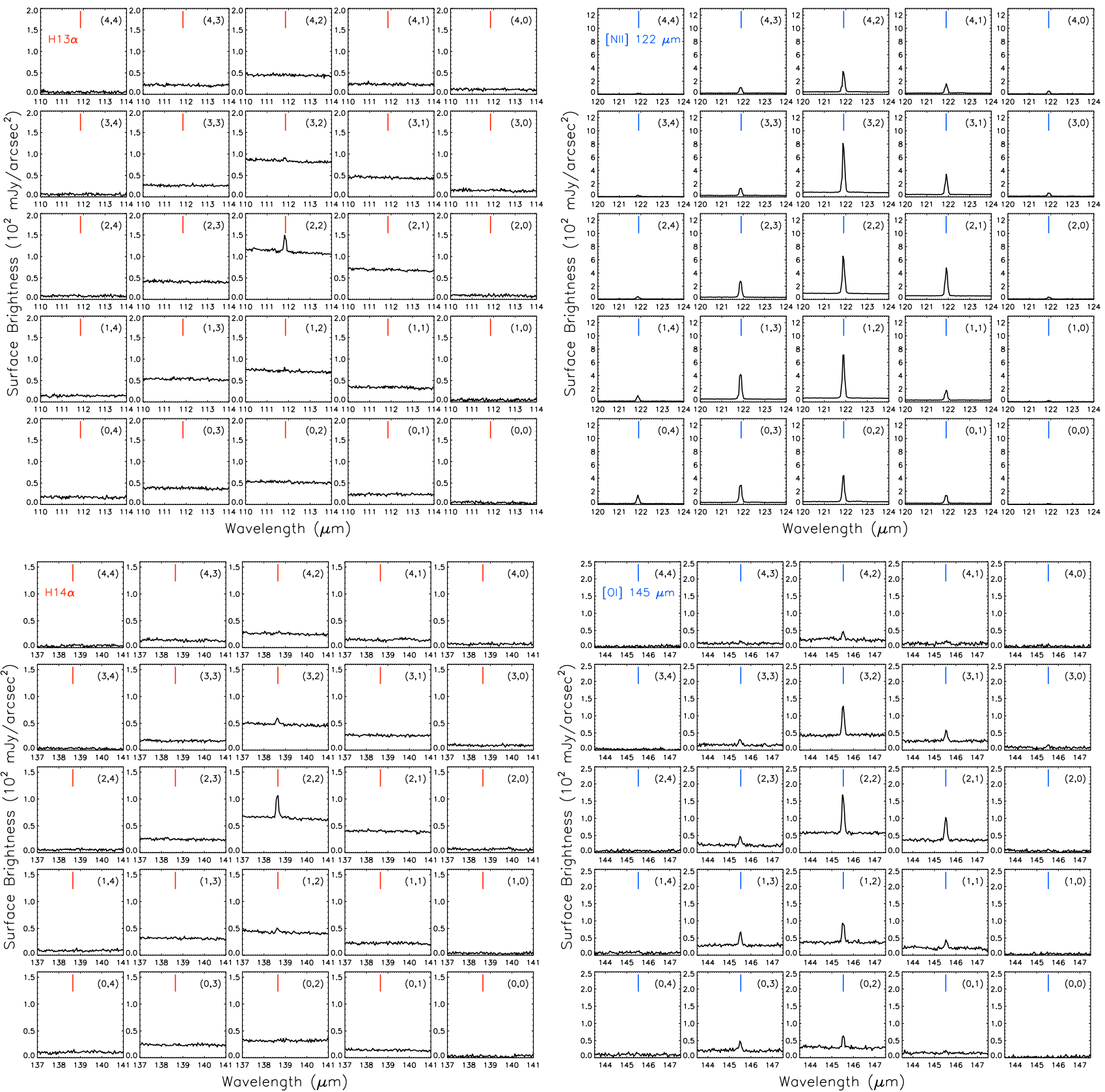

Figure 4. Same as Fig. 3, for different lines.

as we will discuss below. Although the $\mathrm{H} \beta$ flux includes the emission from the whole nebula, the $\mathrm{H} \alpha$ to $\mathrm{H} \beta$ ratios we used only probe the emission within slits widths from $1.5^{\prime \prime}$ to $2.0^{\prime \prime}$. The $\mathrm{H} \alpha$ to $\mathrm{H} \beta$ ratios should however be representative, since the slits cover regions where the emission significantly contributes to the total emission.

The optical to MIR HRL emission of regular PNe and H II Regions can usually be well fitted by the calculated emission for an optically thin gas dominated by spontaneous emission (Osterbrock \& Ferland 2006). We attempted to fit the $\mathrm{Mz} 3$ observed HRL ratios curve using the emissivities provided by Storey \& Hummer (1995). We explored coefficients for the whole range of physical conditions (temperature and density) provided by the authors, but found no reasonable curve that simultaneously fits all the observations (optical to submm).

Mz 3 and MWC 349A HRLs flux ratios are similar. In Fig. 7, we include the MWC 349A HRL ratios (Thum et al. 1998) for comparison. MWC 349A is a massive B[e]SG star and the first object where HRL laser has been detected (Martin-Pintado et al. 1989). As in Mz 3, MWC 349A has a circumstellar bipolar nebula produced by intense outflows and a dense disc seen nearly edge-on in its compact central core (Danchi et al. 2001).

Although the $\mathrm{Mz} 3$ data are not as comprehensive as for MWC 349A in terms of $n$ coverage, we can see that the behaviour of the ratios of both objects are very similar. 

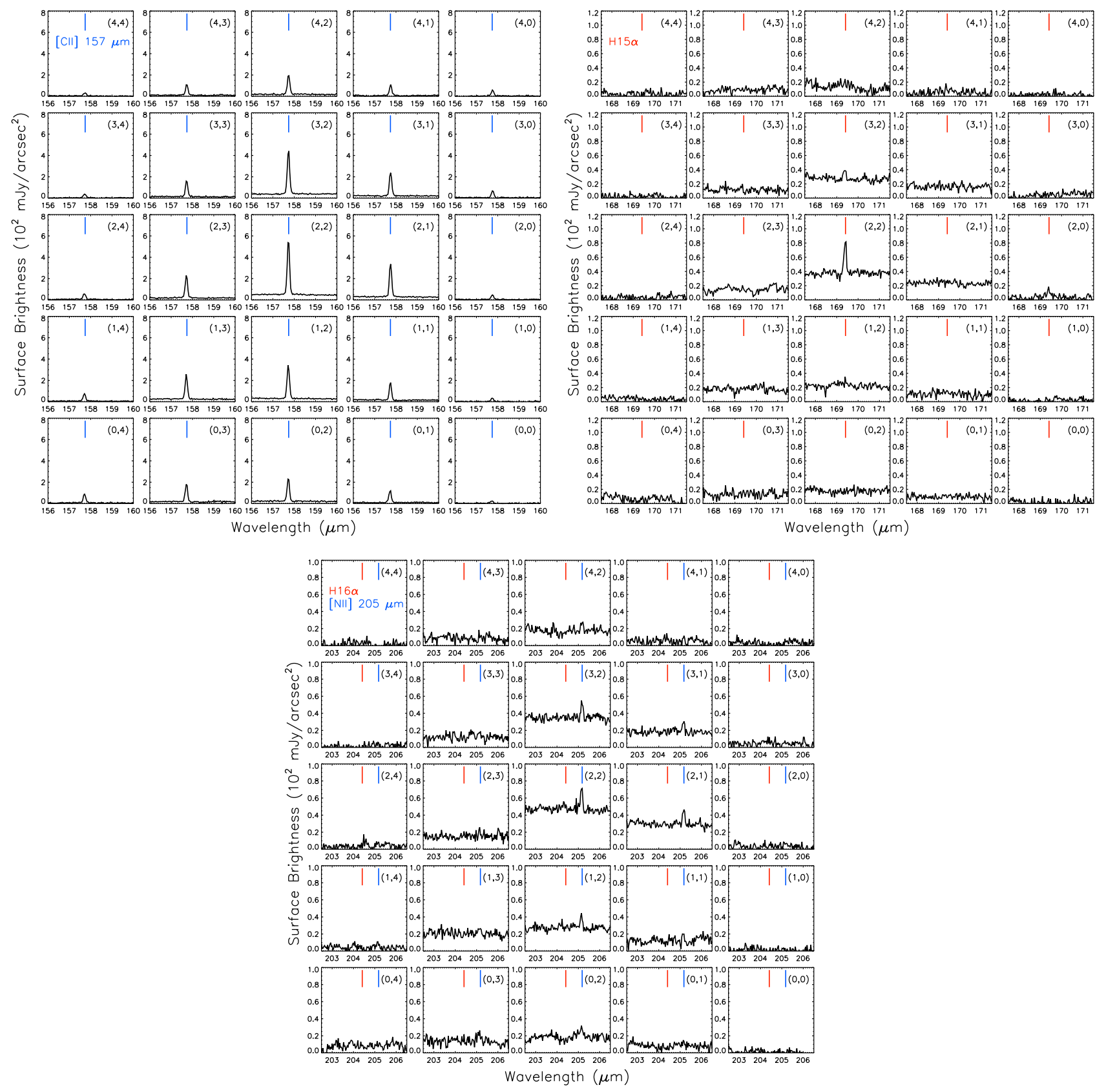

Figure 5. Same as Fig. 3, for different lines.

Strelnitski et al. (1996b) and Thum et al. (1998) studied the behaviour of the $\mathrm{H} n \alpha$ as a function of $n$ for MWC 349A. For $n<6-7$ (optical to MIR), the curve is steeper than the curve expected for the spontaneous emission of an optically thin gas, which indicates that the lines are at least partially optically thick (see Thum et al. 1998, and references therein). A curve with a $n^{-6}$ dependence is expected in this case (Strelnitski et al. 1996b). In the radio regime $(n>40)$, the free-free opacity is important and the HRL ratios must follow a $n^{-8}$ behaviour (assuming a spherically symmetric, constant velocity outflow at $T_{\mathrm{e}}=10^{4} \mathrm{~K}$; Strelnitski et al. 1996b). The theoretical curves in Fig. 7 representing the ratios in both regimes is connected by a curve with a $n^{-7}$ dependence following Strelnitski et al. (1996b). It is clear from Fig. 7 that the data points for MZ 3 follow those of MWC 349A.

In the optical to MIR regime and the radio regime, the HRL emission of MWC 349A follows the solid curve. The MWC 349A FIR to mm lines, on the other hand, clearly deviate from the solid curve. The extensive dataset of MWC 349A allowed Strelnitski et al. (1996b) to show that such enhancement in the emission for $n$ in the interval 8 to 40 is due to amplification by stimulated emission (laser effect) occurring in its core (see also Thum et al. 1998; Báez-Rubio et al. 2013). The HRL laser emission detected in MWC 349A is believed to be produced in the ionized surface layer of its evaporating disc (Thum et al. 1994; Báez-Rubio et al. 2014). 


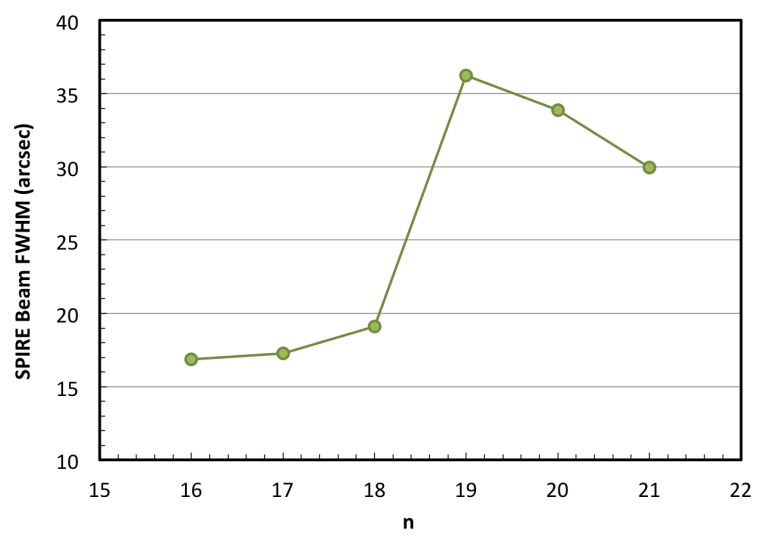

Figure 6. Herschel/SPIRE beam size (full width at half maximum) for the wavelengths of each $\mathrm{H} n \boldsymbol{\alpha}$ line observed in $\mathrm{Mz} 3$.

MWC 349A has been extensively studied and the action of laser effect on its HRLs is well established.

The laser emission is produced in the core of $\mathrm{Mz} 3$. In the previous section, we show that the HRL emission detected in the PACS observations of $\mathrm{Mz} 3$ is concentrated mostly in the central spaxel. Similar evidence is found in the SPIRE lobe pointing observations, as we will discuss in the following.

In the bottom panel of Fig. 7, we provide HRL ratios derived from the SPIRE central bolometer spectrum of the southern lobe instead of the SPIRE central pointing. Although the comparison with the PACS three-spaxels central region is not strictly correct as we are not probing the same region, it is informative and supports the idea that the laser effect is produced in the core and not in the lobes. As for the case of the top panel in Fig. 7, no correction for the different beam sizes was made. In the figure, we can see that the SPIRE line ratios decrease towards the ratios not influenced by laser effect (solid curve) for low $n$ values. This behaviour is due to the decrease of the SPIRE beam size at shorter wavelengths (i.e. for lower $n$ values; see Fig. 6 ). As the beam size decreases, the core contribution to the emission inside the beam also decreases, and the line ratios approach the 'non-laser' values. This behaviour is consistent with the scenario above where the core region is responsible for the laser emission.

Mz 3 and MWC 349A core conditions are similar. MWC 349A has been extensively studied in the literature. Its evolutionary phase is, however, still under debate. This $\mathrm{B}[\mathrm{e}]$ star shows an ionized bipolar structure identified on scales from 0.05 to $1.5 \mathrm{pc}$ as shown by Tafoya et al. (2004) and Gvaramadze \& Menten (2012). In the waist of this bipolar nebula, there is a very compact core comprised of a a central source that ionizes the surface of the surrounding disc. The central source is likely a close binary system (Gvaramadze \& Menten 2012). The secondary appears to be a lowmass star and the primary a massive $\mathrm{B} 0$ or late $\mathrm{O}$ star with stellar parameters in the following ranges: $T_{\mathrm{eff}}=20-30 \mathrm{kK}$, $L_{\star}=3 \times 10^{4}-8 \times 10^{5} L_{\odot}, M=30-40 M_{\odot}$ (Hofmann et al. 2002; Gvaramadze \& Menten 2012, and references therein). The bipolar structure is formed by the strong winds produced at
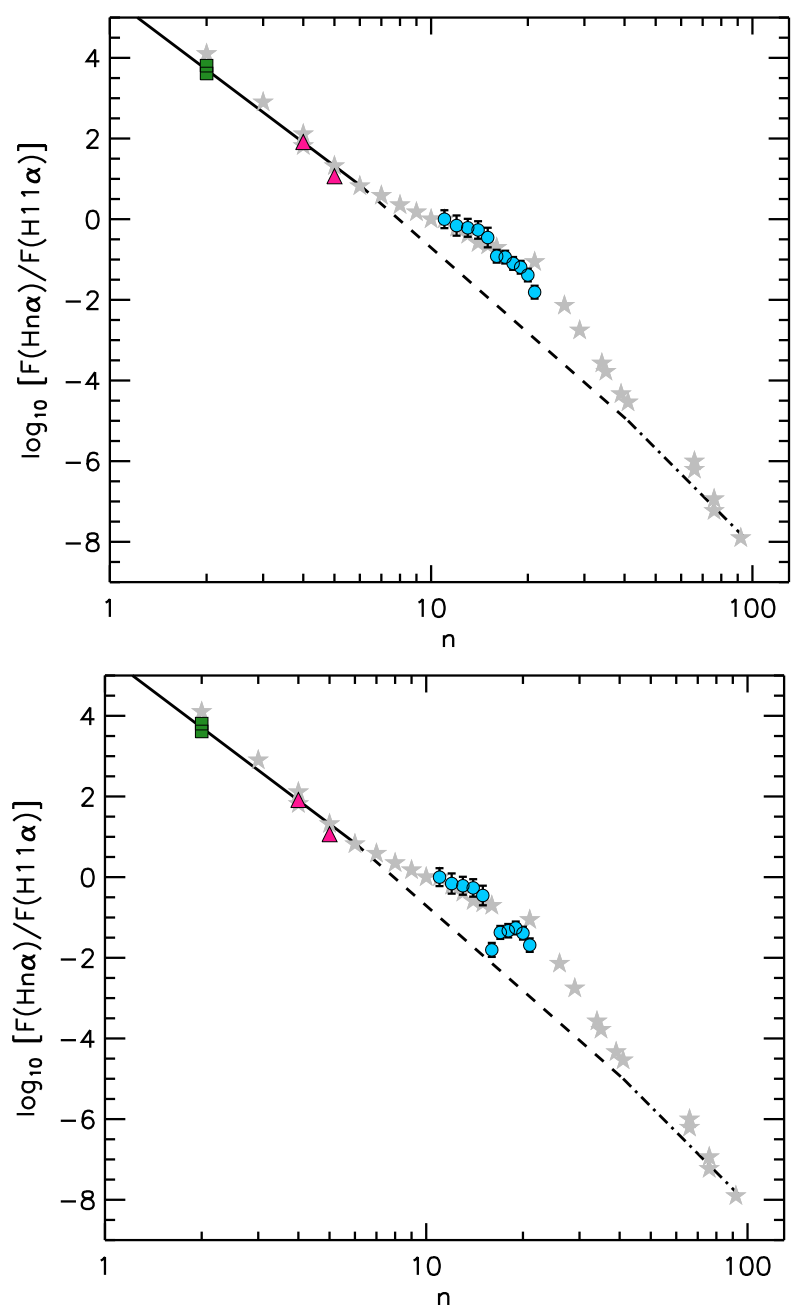

Figure 7. Hn $\alpha$ lines in the Mz 3 FIR/submm spectrum. Mz 3 Herschel observations are represented by dots (this work). PACS fluxes are the values integrated in the three central spaxels. SPIRE fluxes are from the central pointing in the top panel and for the lobe pointing in the bottom panel (central bolometer only for both cases). In both panels, ISO observations of $\operatorname{Br} \alpha$ and $\operatorname{Pf} \alpha$ are represented by triangles (Sloan et al. 2003, this work), and $\mathrm{H} \alpha$ observations of ESO 1.52 and CTIO $1.5 \mathrm{~m}$ optical telescopes by squares (Zhang \& Liu 2002; Smith 2003). Gray stars are observations of MWC 349A compiled by Thum et al. (1998). The solid, dashed, and dot-dashed curves are the theoretical expectation for the nebular spontaneous emission for the optical to MIR, FIR to millimetre, and radio regimes as discussed in Sect. 5 .

the rotating disc surface at radii smaller than 24 AU (BáezRubio et al. 2014). The outflows have velocities of a few tens of $\mathrm{km} \mathrm{s}^{-1}$ (Martín-Pintado 2002; Báez-Rubio et al. 2014). The inclination of the bipolar structure with respect to the plane-of-sky is small $\left(\sim 15^{\circ}\right)$, which indicates that the disc is also seen almost edge-on (Rodriguez \& Bastian 1994). The best model for MWC 349A found by Báez-Rubio et al. (2013) yields an inclination of $8^{\circ}$ with respect to the lineof-sight for the disc. The size of the disc is estimated to be roughly between 50 to $120 \mathrm{AU}$ (Sallum et al. 2017). The inner radius should be less than 3 AU (Báez-Rubio et al. 2013).

According to Thum et al. (1994), the characteristics of 
the submm lines "can be explained if the masers are on the ionized surface of a rotating disc with an additional small velocity component direct toward the center of rotation". The fact that the disc is seen edge-on is significant, as this will maximise the laser amplification length along the emitting surface. It is only necessary that the disc is seen roughly edge-on to have a sufficient effect (Thum et al. 1998). In MWC 349A, laser effect has been detected in HRLs with $n$ between 10 and 40 (MIR to radio; Strelnitski et al. 1996b; Thum et al. 1998; Martín-Pintado 2002; Báez-Rubio et al. 2013). Calculations by Hengel \& Kegel (2000) and Calculations by Hengel \& Kegel (2000) and Thum et al. (1998) indicated that the densities in the zones where the laser lines are produced are $\sim 10^{8} \mathrm{~cm}^{-3}$.

The structure and physical conditions of the core of $\mathrm{Mz} 3$ is very similar to those of MWC 349A. Mz 3 exhibits a bipolar structure with a very compact core where a nearly edge-on disc surrounds the ionizing central source. Santander-García et al. (2004) inferred that the lobes of $\mathrm{Mz} 3$ are inclined in $\sim 17^{\circ}$ in relation to the plane-of-sky. Outflows velocities between 130 and $500 \mathrm{~km} \mathrm{~s}^{-1}$ have been observed (Santander-García et al. 2004; Redman et al. 2000).

Photoionization analysis indicates that the ionizing source of Mz 3 has $T_{\text {eff }}=39500 \mathrm{~K}$ and $L_{\star}=2300-10000 L_{\odot}$ (Pottasch \& Bernard-Salas 2010; Smith 2003). However, the presence of a large flux of hard X-Rays $(\mathrm{E}>1 \mathrm{keV})$ in the core seems to indicate that the central source may be even more luminous, but deeply embedded by X-ray absorbing material (Kastner et al. 2003). This is in line with the high extinction estimated for the $\mathrm{Mz} 3$ core previously mentioned.

Using optical iron diagnostic line ratios, Zhang \& Liu (2002) determined that the iron emission observed from the core of $\mathrm{Mz} 3$ is emitted by a region with densities around $10^{6.5} \mathrm{~cm}^{-3}$. Smith (2003) used the observed suppression of a few atomic forbidden lines in the core and the concept of critical densities to estimate that the core region must have densities in excess of $10^{6}-10^{7} \mathrm{~cm}^{-3}$. In both cases, the authors made clear that regions with densities above the forbidden lines critical densities may still exist, but can not be probed by such lines. Chesneau et al. (2007) obtained a model for the $\mathrm{Mz} 3$ disc, which was recently improved by Macdonald et al. (2017). According to those works, the disc is rather flat, seen nearly edge-on and rich in amorphous silicates. The dust mass in the core is $2 \times 10^{-5} M_{\odot}$ (Gesicki et al. 2010). With a inner and outer disk radius of $\sim 10 \mathrm{AU}$ and $\sim 250 \mathrm{AU}$, respectively, and a disk thickness on the order of $10 \mathrm{AU}$, an average density of $2 \times 10^{8} \mathrm{~cm}^{-3}$ is obtained if a dust-to-gas ratio of 100 is assumed. This is a rough approximation, but indicates a core density similar to that at MWC 349A and a density necessary for the production of FIR/submm laser lines exist.

Zhang et al. (2012) found many similarities between the Mz 3 and NGC 2392 structures and abundances, which made the authors propose that both PNe have a similar origin in a binary system where a giant star is responsible for the central outflows being ejected inside the planetary nebula previously produced by its now compact companion. NGC 2392 has a structure very similar to $\mathrm{Mz} 3$, but it is seen almost pole-on, which can explain the absence of $\mathrm{H}$ I laser lines in the NGC 2392 Herschel spectrum. Furthermore, Zhang et al. (2012) have not found evidence of very high density regions in the core of NGC 2392.
We cannot determine the exact extent of the $n$ range where laser is acting in $\mathrm{Mz} 3$ due to the lack measurements at MIR, short wavelength FIR, and mm ranges (Fig. 7). However, if the low- $n$ onset of laser amplification is close to $n=8$ 10 as for MWC 349A, which seems reasonable from Fig. 7, the density of the emitting gas could reach $10^{8}-10^{10} \mathrm{~cm}^{-3}$ in the Mz 3 core (Strelnitski et al. 1996a). The existence of densities exceeding $10^{6} \mathrm{~cm}^{-3}$ were determined by for $\mathrm{Mz} 3$ Smith (2003) from optical emission.

Considering the similarities in the HRL emission of Mz 3 and MWC 349A and in the relevant physical structure and conditions of their cores as discussed above, it is natural to expect that laser effect may take place in the $\mathrm{Mz} 3$ core. It is then reasonable to conclude that laser effect is a likely explanation to the detection and the observed enhancement in the FIR/submm HRL intensity ratios we observed in the core of $\mathrm{Mz} 3$ with Herschel.

The discovery of laser effect acting on the FIR/submm HRL emission puts $\mathrm{Mz} 3$ on a very short list of objects where HRL lasers have been detected. Table 3 lists those objects and some of their properties. Besides MWC 349, it has been shown that HRL lasers occur in MWC 922, $\eta$ Carinae, Cepheus A HW2, MonR2-IRS2, and M 82. The list includes objects of very different classes: one PN/Symbiotic, two $\mathrm{B}[\mathrm{e}]$ stars, a blue luminous variable, two young stellar objects, and a galaxy. However, all the objects have in common a strong ionized bipolar outflow and a dense disc structure associated with the HRL laser-emitting region.

\section{CONCLUSIONS}

In this paper, we report the detection of hydrogen recombination laser lines in the FIR to submm spectrum of $\mathrm{Mz} 3$ observed with the Herschel PACS and SPIRE instruments. Comparison of optical to submm HRL lines to theoretical calculations indicates that there is an enhancement in the FIR to submm HRLs, which explains their unexpected detection. The likely explanation for this enhancement is the occurrence of laser effect. Laser effect offers a natural explanation since:

(i) the available $\mathrm{Mz} 3$ optical to submm HRL $\alpha$ line intensity ratios are not well reproduced by the spontaneous emission of an optically thin ionized gas, which is typical for the nebular gas in planetary nebula;

(ii) the compact core is responsible for a large fraction of the Mz 3 Herschel HRLs emission;

(iii) the line intensity ratios for $\mathrm{Mz} 3$ are very similar to those in the core emission of the notorious star MWC 349A, where it is well established that laser effect is responsible for the enhancement of HRLs in the Herschel wavelength range;

(iv) the physical characteristics in the core MWC 349A that are responsible for producing the conditions for laser effect, i.e. dense equatorial disc seen nearly edge-on and intense ionized outflows, are also present in the Mz 3 core.

Our comparison of observations to models of laser emission from the literature indicates the presence of a dense and ionized gas $\left(n_{\mathrm{H}}>10^{8} \mathrm{~cm}^{-3}\right)$ in the core of $\mathrm{Mz} 3$. For the surrounding lobes, the empirical analysis of forbidden lines indicates densities around $4500 \mathrm{~cm}^{-3}$.

We have presented compelling evidence to support that 
Table 3. Properties of Objects with H Laser Emission

\begin{tabular}{|c|c|c|c|c|c|c|c|}
\hline Object & $\begin{array}{l}\text { Ref. Detection } \\
\text { of } \mathrm{H} \text { laser lines }\end{array}$ & Classification I $^{a}$ & Classification $\mathrm{II}^{b}$ & $\begin{array}{l}\text { Strong Winds/ } \\
\text { Outflows }\end{array}$ & $\begin{array}{l}\text { Outflow } \\
\text { Morphology }\end{array}$ & Disc & Ref. $^{c}$ \\
\hline $\mathrm{Mz} 3$ & 1 & Planetary Nebula & $\begin{array}{l}\text { Young PN } \\
\text { pre-PN } \\
\text { Symbiotic }\end{array}$ & Yes & Bipolar & Yes & $2-8$ \\
\hline MWC 349A & 9 & Emission-line Star & $\begin{array}{c}\text { B[e]SG star } \\
\text { Luminous Blue Variable }\end{array}$ & Yes & Bipolar & Yes & $10-14$ \\
\hline MWC 922 & 15 & Emission-line Star & B[e] FS CMa star & Yes & Bipolar & Yes & $15-16$ \\
\hline$\eta$ Car & 17 & Emission-line Star & Luminous Blue Variable & Yes & Bipolar & Yes & $17-19$ \\
\hline Cep A HW2 & 20 & Young Stellar Object & - & Yes & Bipolar & Yes & $20-22$ \\
\hline MonR2-IRS2 & 23 & Star in Cluster & $\begin{array}{l}\text { Compact YSO } \\
\text { TTauri Star }\end{array}$ & Yes & Bipolar & Yes & $23-24$ \\
\hline M82 & 25 & Interacting Galaxies & Starburst Galaxy & Yes & Bipolar & Yes & $25-26$ \\
\hline
\end{tabular}

Notes: ${ }^{a}$ Classification from CDS/Simbad. ${ }^{b}$ Classification from other sources and complementing data (see references in the last column). ${ }^{c}$ References for data in columns 4 to 7 .

References: (1) This Work; (2) Cohen et al. (1978); (3) Lopez \& Meaburn (1983); (4) Schmeja \& Kimeswenger (2001);

(5) Bains et al. (2004); (6) Cohen et al. (2011); (7) Meaburn \& Walsh (1985); (8) Chesneau et al. (2007);

(9) Martin-Pintado et al. (1989); (10) Aret et al. (2016); (11) White \& Becker (1985); (12) Gvaramadze \& Menten (2012);

(13) Thum et al. (1994); (14) Martín-Pintado et al. (2011); (15) Sánchez Contreras et al. (2017); (16) Wehres et al. (2017);

(17) Cox et al. (1995); (18) Meaburn et al. (1993); (19) Abraham et al. (2014); (20) Jiménez-Serra et al. (2011);

(21) Jiménez-Serra et al. (2007); (22) Sugiyama et al. (2014); (23) Jiménez-Serra et al. (2013); (24) Carpenter \& Hodapp (2008);

(25) Seaquist et al. (1996); (26) Rodriguez-Rico et al. (2004)

laser effect is acting on the HRLs in Mz 3. Future work will improve the coverage of HRLs from $\mathrm{Mz} 3$ to complete the curve shown in Fig. 7, as well as, resolved line velocity profiles. Velocity profiles can be used to constrain detailed models of the core structure of Mz 3. Sub-arcsecond submm ALMA observations would be ideal to isolate the central region from the rest of the nebula and resolve the laser components.

\section{ACKNOWLEDGEMENTS}

I.A. acknowledges partial financial support of $\mathrm{CNPq} /$ Brazil. Studies of interstellar chemistry at Leiden Observatory are supported through the advanced-ERC grant 246976 from the European Research Council, through a grant by the Dutch Science Agency, NWO, as part of the Dutch Astrochemistry Network, and through the Spinoza prize from the Dutch Science Agency, NWO. S.W. acknowledges the Leiden/ESA Astrophysics Program for Summer Students (LEAPS), which supported his visit to the Leiden Observatory. P.v.H. is supported by the Belgian Federal Science Policy Office under contract No. BR/143/A2/BRASS. MO was supported by the research fund 106-2811-M-001-119 and 104-2112-M001-041-MY3 from the Ministry of Science and Technology (MOST), R.O.C. We thank S. Akras for useful discussions about Mz 3. This research has made use of NASA's Astrophysics Data System and the SIMBAD database, operated at CDS, Strasbourg, France. Some of the data presented in this paper were obtained from the Mikulski Archive for Space Telescopes (MAST). STScI is operated by the Association of Universities for Research in Astronomy, Inc., under NASA contract NAS5-26555. Support for MAST for nonHST data is provided by the NASA Office of Space Science via grant NNX09AF08G and by other grants and contracts.

\section{REFERENCES}

Abraham Z., Falceta-Gonçalves D., Beaklini P. P. B., 2014, ApJ, 791,95

Aret A., Kraus M., Šlechta M., 2016, MNRAS, 456, 1424

Báez-Rubio A., Martín-Pintado J., Thum C., Planesas P., 2013, A\&A, 553, A45

Báez-Rubio A., Martín-Pintado J., Thum C., Planesas P., TorresRedondo J., 2014, A\&A, 571, L4

Bains I., Redman M. P., Bryce M., Meaburn J., 2004, MNRAS, 354,549

Bujarrabal V., Bachiller R., 1991, A\&A, 242, 247

Carpenter J. M., Hodapp K. W., 2008, The Monoceros R2 Molecular Cloud. p. 899

Chesneau O., et al., 2007, A\&A, 473, L29

Clyne N., Akras S., Steffen W., Redman M. P., Gonçalves D. R., Harvey E., 2015, A\&A, 582, A60

Cohen M., Kunkel W., Lasker B. M., Osmer P. S., Fitzgerald M. P., 1978, ApJ, 221, 151

Cohen M., Parker Q. A., Green A. J., Miszalski B., Frew D., Murphy T., 2011, MNRAS, 413, 514

Corradi R. L. M., et al., 2010, A\&A, 509, A41

Cox P., Martin-Pintado J., Bachiller R., Bronfman L., Cernicharo J., Nyman L.-A., Roelfsema P. R., 1995, A\&A, 295, L39

Danchi W. C., Tuthill P. G., Monnier J. D., 2001, ApJ, 562, 440

Gesicki K., Zijlstra A. A., Szyszka C., Hajduk M., Lagadec E., Guzman Ramirez L., 2010, A\&A, 514, A54

Griffin M. J., et al., 2010, A\&A, 518, L3

Guerrero M. A., Chu Y.-H., Miranda L. F., 2004, AJ, 128, 1694

Gvaramadze V. V., Menten K. M., 2012, A\&A, 541, A7

Hengel C., Kegel W. H., 2000, A\&A, 361, 1169

Hofmann K.-H., Balega Y., Ikhsanov N. R., Miroshnichenko A. S., Weigelt G., 2002, A\&A, 395, 891

Huggins P. J., Bachiller R., Cox P., Forveille T., 1996, A\&A, 315, 284

Jiménez-Serra I., Martín-Pintado J., Rodríguez-Franco A., Chandler C., Comito C., Schilke P., 2007, ApJ, 661, L187

Jiménez-Serra I., Martín-Pintado J., Báez-Rubio A., Patel N., Thum C., 2011, ApJ, 732, L27

Jiménez-Serra I., Báez-Rubio A., Rivilla V. M., Martín-Pintado J., Zhang Q., Dierickx M., Patel N., 2013, ApJ, 764, L4 
Kastner J. H., Balick B., Blackman E. G., Frank A., Soker N., Vrtílek S. D., Li J., 2003, ApJ, 591, L37

Kaufman M. J., Wolfire M. G., Hollenbach D. J., 2006, ApJ, 644, 283

Kingsburgh R. L., English J., 1992, MNRAS, 259, 635

Kramida A., Yu. Ralchenko Reader J., and NIST ASD Team 2017, NIST Atomic Spectra Database (ver. 5.5.1), [Online]. Available: https://physics.nist.gov/asd [2017, December 8]. National Institute of Standards and Technology, Gaithersburg, MD.

Lee T.-H., Lim J., Kwok S., 2007, ApJ, 665, 341

Liu X.-W., et al., 1996, A\&A, 315, L257

Liu X.-W., et al., 2001, MNRAS, 323, 343

Lopez J. A., Meaburn J., 1983, MNRAS, 204, 203

Macdonald D., De Marco O., Lagadec E., Ma J., Chesneau O., 2017, preprint, (arXiv: 1705.00120)

Makiwa G., Naylor D. A., Ferlet M., Salji C., Swinyard B., Polehampton E., van der Wiel M. H. D., 2013, Appl. Opt., 52, 3864

Markwardt C. B., 2009, in Bohlender D. A., Durand D., Dowler P., eds, Astronomical Society of the Pacific Conference Series Vol. 411, Astronomical Data Analysis Software and Systems XVIII. p. 251 (arXiv:0902.2850)

Martín-Pintado J., 2002, in Migenes V., Reid M. J., eds, IAU Symposium Vol. 206, Cosmic Masers: From Proto-Stars to Black Holes. p. 226

Martin-Pintado J., Bachiller R., Thum C., Walmsley M., 1989, A\&A, 215, L13

Martín-Pintado J., Thum C., Planesas P., Báez-Rubio A., 2011, A\&A, 530, L15

Meaburn J., Walsh J. R., 1985, MNRAS, 215, 761

Meaburn J., Walsh J. R., Wolstencroft R. D., 1993, A\&A, 268, 283

Menzel D. N., 1922, Harvard College Observatory Bulletin, 777, 0

Menzel D. H., 1937, ApJ, 85, 330

Moré J. J., 1978, in Watson G. A., ed., Numerical Analysis: Proceedings of the Biennial Conference Held at Dundee, June 28-July 1, 1977. Springer Berlin Heidelberg, Berlin, Heidelberg, pp 105-116, doi:10.1007/BFb0067700, http://dx.doi. org/10.1007/BFb0067700

Osterbrock D. E., Ferland G. J., 2006, Astrophysics of gaseous nebulae and active galactic nuclei. University Science Books

Otsuka M., et al., 2017, ApJS, 231, 22

Ott S., 2010, in Mizumoto Y., Morita K.-I., Ohishi M., eds, Astronomical Society of the Pacific Conference Series Vol. 434, Astronomical Data Analysis Software and Systems XIX. p. 139

Pilbratt G. L., et al., 2010, A\&A, 518, L1

Poglitsch A., et al., 2010, A\&A, 518, L2

Pottasch S. R., Bernard-Salas J., 2010, A\&A, 517, A95

Pottasch S. R., Surendiranath R., 2005, A\&A, 444, 861

Pound M. W., Wolfire M. G., 2008, in Argyle R. W., Bunclark P. S., Lewis J. R., eds, Astronomical Society of the Pacific Conference Series Vol. 394, Astronomical Data Analysis Software and Systems XVII. p. 654

Redman M. P., O'Connor J. A., Holloway A. J., Bryce M., Meaburn J., 2000, MNRAS, 312, L23

Rodriguez L. F., Bastian T. S., 1994, ApJ, 428, 324

Rodriguez-Rico C. A., Viallefond F., Zhao J.-H., Goss W. M., Anantharamaiah K. R., 2004, ApJ, 616, 783

Sallum S., Eisner J. A., Hinz P. M., Sheehan P. D., Skemer A. J., Tuthill P. G., Young J. S., 2017, ApJ, 844, 22

Sánchez Contreras C., Báez-Rubio A., Alcolea J., Bujarrabal V., Martín-Pintado J., 2017, A\&A, 603, A67

Santander-García M., Corradi R. L. M., Balick B., Mampaso A., 2004, A\&A, 426, 185

Schmeja S., Kimeswenger S., 2001, A\&A, 377, L18

Seaquist E. R., Carlstrom J. E., Bryant P. M., Bell M. B., 1996,
ApJ, 465, 691

Sloan G. C., Kraemer K. E., Price S. D., Shipman R. F., 2003, ApJS, 147, 379

Smith N., 2003, MNRAS, 342, 383

Storey P. J., Hummer D. G., 1995, MNRAS, 272, 41

Strelnitski V. S., Ponomarev V. O., Smith H. A., 1996a, ApJ, 470, 1118

Strelnitski V. S., Smith H. A., Ponomarev V. O., 1996b, ApJ, 470,1134

Sugiyama K., et al., 2014, A\&A, 562, A82

Swinyard B. M., et al., 2010, A\&A, 518, L4

Tafoya D., Gómez Y., Rodríguez L. F., 2004, ApJ, 610, 827

Thum C., Matthews H. E., Martin-Pintado J., Serabyn E., Planesas P., Bachiller R., 1994, A\&A, 283, 582

Thum C., Martin-Pintado J., Quirrenbach A., Matthews H. E., 1998, A\&A, 333, L63

Ueta T., et al., 2014, A\&A, 565, A36

Wehres N., Ochsendorf B. B., Tielens A. G. G. M., Cox N. L. J., Kaper L., Bally J., Snow T. P., 2017, A\&A, 601, A69

Wesson R., et al., 2010, A\&A, 518, L144

White R. L., Becker R. H., 1985, ApJ, 297, 677

Zhang Y., Liu X.-W., 2002, MNRAS, 337, 499

Zhang Y., Fang X., Chau W., Hsia C.-H., Liu X.-W., Kwok S., Koning N., 2012, ApJ, 754, 28

Škoda P., Draper P. W., Neves M. C., Andrešič D., Jenness T., 2014, Astronomy and Computing, 7, 108

This paper has been typeset from a $\mathrm{T}_{\mathrm{E} X} / \mathrm{LAT}_{\mathrm{E}} \mathrm{X}$ file prepared by the author. 